\title{
Optimizing the Output of a Human-Powered Energy Harvesting System with Miniaturization and Integrated Control
}

\author{
Vishwa Goudar*, Zhi Ren ${ }^{\dagger}$, Paul Brochu ${ }^{\dagger}$, Miodrag Potkonjak* and Qibing Pei ${ }^{\dagger}$ \\ ${ }^{*}$ Computer Science Department, University of California, Los Angeles \\ $\dagger$ Department of Material Science and Engineering, University of California, Los Angeles
}

\begin{abstract}
We propose a novel harvesting technology to inconspicuously transduce mechanical energy from human foot-strikes and explore its configuration and control towards optimized energy output. Dielectric Elastomers (DEs) are high-energy density, soft, rubber-like material that electrostatically transduce mechanical energy. These properties enables increased energy-transduction efficiency without sacrificing on user comfort, if configured and controlled properly. We expose key statistical properties of human gait which show that an array of miniaturized harvesters across the foot-sole will improve energy output. Further, the gait properties naturally yield a closed-loop control strategy to individually control harvesters in the array in a manner that maximizes net energy output. We propose statistical techniques that guide the configuration and control of the harvester array, and evaluate system behavior from detailed analytical and empirical models of DE behavior. System evaluations based on experimentally collected foot pressure datasets from multiple subjects show that the proposed system can achieve up to $120 \mathrm{~mJ}$ per foot-strike, enough to power a variety of low-power wearable devices and systems.
\end{abstract}

\section{INTRODUCTION}

Recent technical innovations in the miniaturization, flexibility and performance of sensors and sensor networks have yielded increasingly well-integrated and connected smart wearable systems. This has resulted in prototypes and commercial designs that are not just promising in terms of the previously-unachievable capabilities that they can deliver to the fields of medicine, environmental monitoring, participatory sensing, human-computer interaction and entertainment, but also in terms of their ability to acutely restrict inconvenience to the user and interference with the fidelity and functionality of the platform that they are integrated into. However, the extent to which their energy demands contribute to diminishing operating lifetimes and extending maintenance overhead has stymied rapid penetration. To alleviate these issues, several energy/power-efficient optimizations have been offered for wearable system design [1], [2].

Aside from reducing power consumption in such systems, harvesting human energy output as a potential energy source has also been explored. In fact, human energy output has been explored as an alternative energy source in various forms (thermal, chemical, kinetic) and from various human actions [3], [4]. For instance, foot strikes have been estimated to yield significant amounts of energy a 154-pound person can produce upwards of $7 \mathrm{~W}$ per foot strike [3]. However, the portion of this that can be scavenged is limited by two factors. First, prevalent energy transduction technologies are limited in their energy densities, and therefore in their capacity for such quantities of energy. Second, the nature of these technologies is such that they are unable to transduce much energy output without causing discomfort to the user. For example, a hydraulic-amplified piezoelectric based foot-strike energy harvesting system was described in [3], capable of producing $675 \mathrm{~mW}$ per foot-strike while adding significant heft to the shoe, thereby altering the user's gait. In contrast, they also described a piezo-electric based foot-strike energy harvesting system that interfered minimally with the user's gait and was capable of producing $10-20 \mathrm{~mW}$.

Recently, a new class of harvesters have emerged with the promise of superior transduction capability and minimal impact on user comfort. Dielectric Elastomers (DEs) are high energy-density rubberlike materials that possess the ability to behave as energy generators, actuators and sensors. Their ability to yield 5 to 40 times the energy density of piezoelectrics [5] makes them more effective transducers. Their soft rubber-like nature further enables near-transparency of the harvesting system to the user. However, while competing technologies transduce ambient energy by exploiting underlying physical phenomena (e.g. seebeck, photovoltaic and piezoelectric effects), DEs transduce mechanical energy based on an electrostatic principle. Hence, their configuration and control is key to the levels of energy that they may harvest.

In this paper, we study the use of DEs to scavenge energy from human foot-strikes and drive low-power wearable systems. Our objective here is to design an energy harvesting platform with maximum energy output under the constraints that the system will be comfortable to the user and that it will abide by power constraints of the target wearable system. From experimentally-collected plantar pressure datasets, we expose 3 key properties commonly observed in human gait, which we leverage to optimize DE configuration and control towards the stated objective: (i) Foot-strikes exhibit spatiotemporal variance with respect to the amount of energy expended across the foot sole and over the duration of the stride. This guides the configuration of a shoe-sole integrated DE harvester design that is comprised of an array of miniaturized harvesters rather than a single sole-wide harvester; (ii) Foot-strikes exhibit spatial variance with respect to the maximum energy expended at different regions of the foot. This influences how aggressive a harvester is, based on its location under the foot - low-energy regions of the sole are targeted by more aggressive harvesters while heeding to user-comfort requirements; (iii) Foot strikes exhibit local correlation with respect to the spatio-temporal profile of energy expended. By leveraging integrated/dual-mode operation of DE's as generators and pressure sensors, and expending system power in collecting pressure samples at a few locations and sampling epochs during the user's stride, we can achieve adaptive, targeted and near-optimal control of individual harvesters in the array thereby enhancing net system output.

We validate our proposed transducer configuration by experimentally characterizing and modeling its behavior in detail. The resulting models, in conjunction with our proposed adaptive control algorithm, are applied to experimentally collected datasets of foot pressure of multiple subjects. We demonstrate that our DE based foot-strike energy harvesting system can yield up to $120 \mathrm{~mJ}$ per footstrike, producing enough energy to independently power a number of wearable platforms [6], [7]. Furthermore, we observe that by leveraging statistical properties of human gait to configure the system as an array of miniaturized harvesters with targeted and individualized control, the system achieves up to a $300 \%$ increase in the net energy harvested.

The rest of this paper is organized as follows. In section II, we discuss related work in DE research. Section III provides a synopsis of properties and transduction principles of DEs, and describes characteristics of human gait that are relevant to harverster design and 
control optimizations. Section IV outlines the design of the DE-based harvester platform, while section V details our mathematical model of DE transduction, including the experimental characterization of its mechanical behavior. In section VI, we detail control techniques to optimize the energy transduced from foot strikes with the DE-based platform, and evaluate these techniques in section VII. Section VIII concludes the paper.

\section{RELATED WORK}

DEs are a relatively new entrant to the class of miniaturized generators. An excellent survey of the material properties relevant to its transduction mechanism, various proposed transducer configurations, capabilities in comparison to other common transducers, recent applications, and, operational boundaries and lifetime issues are detailed in [5]. However, due to its basis in electrostatic transduction, maximizing the output of a DE generator, strongly relies on the transducer configuration and control methodology. We also note that DEs require charging at high voltage so they may achieve their output potential; However self-priming circuits have been proposed that use an inverse charge pump to convert some of the DE voltage boost into charge, incrementally increasing the source voltage from $10 \mathrm{~V}$ to the $\mathrm{kV}$ range [8]. Finally, adaptive control of DEs has been proposed in the context of actuation [9], however to the best of our knowledge, we are the first to propose an energy-maximizing adaptive control technique for DE generators.

\section{PRELIMINARIES}

\section{A. Dielectric Elastomer Generators}

DEs are deformable yet incompressible insulating polymer films with high electric permittivity $\left(\varepsilon_{r}\right)$ and relatively low mechanical and electrical losses. This leads naturally to their use as variable capacitors capable of direct transduction of mechanical to electrical energy, with little need for additional mechanical components and complex structure. Their relatively high elastic energy density (5 to 40 times that of piezoelectrics) makes them extremely productive as transducers as they can store more energy when deformed for the same mass and volume of transducer material [5]. At the same time, they are quite soft compared to piezoelectrics, making them less intrusive for human use. Together, these properties endow them with the potential to scavenge human energy more efficiently, while being incorporated into wearable items in low-complexity configurations that are near-transparent to the user.

Typically, DEs are operated as a generator in the constant voltage regime or the constant charge regime. However, it has been analytically shown that the constant voltage regime leads to a lower net energy output, when all other factors are held the same. The operation of a DE in the constant charge regime occurs in 4 stages (Fig. 1). In the actuation stage, the DE is stretched by the mechanical pressure applied to it thereby increasing its capacitance. In the charging stage, electrical charge is added to the elastomer's surface at a fixed voltage $V$, yielding a charge proportional to its capacitance. Throughout the transduction stage that follows, the charge $Q$ on the DE surface is held constant. Here, as the applied mechanical pressure relaxes to zero, the elastic force in the DE are transduced into electrostatic force. As the DE relaxes and changes shape, its capacitance decreases leading to an increase in its voltage and the electrical energy it stores. Over the discharging stage, the voltage discharged by the DE is rectified and the resulting energy added to the battery.

The relationship between a DEs capacitance and stretch is crucial to its net energy output, and can be characterized as follows. If the length and width of the elastomer film each increase by a factor of $\lambda$ when stretched, the area $(A)$ will have increased by a factor of

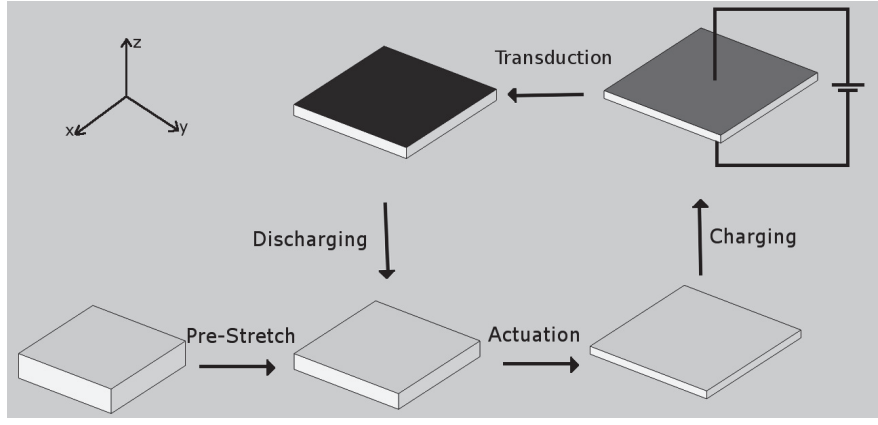

Fig. 1: Dielectric elastomer generator operation cycle.

$\lambda^{2}$. As an incompressible material, the volume must stay constant to cause a decrease in thickness $(d)$ by a factor of $\lambda^{2}$. Equations (1a) and (1b) show that this yields an increase in the capacitance of the DE, from $C_{0}$ in the un-stretched state to $C_{\lambda}$ in the stretched state, by a factor of $\lambda^{4}$. Here, $\varepsilon_{0}$ is vacuum permittivity. The reason behind the high energy density of DEs is underscored by equations (1b) thru (1d), where $\Delta E_{D E}$ is the DE's net energy output, $\lambda_{\text {init }}$ is the stretch in the DE when charge is applied, and $\lambda_{\text {final }}$ is the stretch in the DE when it is discharged. If it is fully relaxed at the end of the transduction stage, the electrical energy in the DE will have increased to a factor of $\lambda^{4}$ of the input electrical energy. This is in contrast to a maximum $\lambda^{2}$ factor increase in energy in conventional electrostatic transducers, a limitation of their rigid structure.

$$
\begin{aligned}
C_{\lambda} & =\frac{\varepsilon_{0} \varepsilon_{r} \lambda^{2} A}{\frac{d}{\lambda^{2}}} \\
& =\lambda^{4} C_{0} \\
\Delta E_{D E} & =\frac{Q^{2}}{2 C_{0}}\left(\frac{1}{\lambda_{\text {final }}^{4}}-\frac{1}{\lambda_{\text {init }}^{4}}\right) \\
& =\frac{C_{\lambda_{\text {init }}} V^{2}}{2}\left(\frac{\lambda_{\text {init }}^{4}}{\lambda_{\text {final }}^{4}}-1\right)
\end{aligned}
$$

\section{B. Human Gait}

Gait is defined as the way in which movement is achieved by humans with their limbs, such as walking, running, hopping, etc. The gait cycle, or stride, is divided into two phases, the stance phase, when the limb is in contact with the ground, and the swing phase, when the limb is in the air for advancement. Although gait characteristics vary across people, human locomotion, but its very nature produces commonalities across people. Fig. 2 illustrates sub-phases of the stance phase along with plantar pressure profiles corresponding to those sub-phases. These profiles are averages over several strides from one of our datasets, collected with a high spatial-resolution plantar pressure monitoring system. They depict the heel at the left and toes at the right, with lighter colors corresponding to higher pressure.

From this figure, three properties of plantar pressure come the fore which generalize across human subjects: (i) Spatio-Temporal Variance - different regions observe maximum pressure at different times; (ii) Spatial Variance - the maximum pressure observed at different regions under the foot is different; and (iii) Local Correlation - the pressure profiles present spatial correlation localized to small regions across the foot sole. We leverage these key properties of human gait

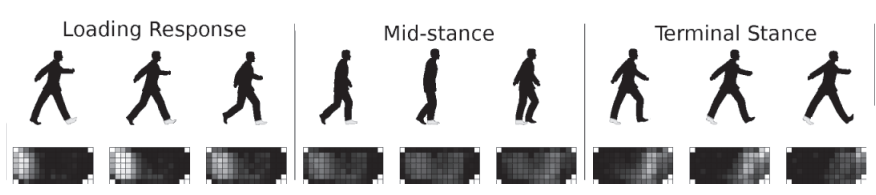

Fig. 2: Foot pressure profiles for sub-phases of stance phase. 
to amplify the energy output of a DE-based harvester system that is based on a miniaturized transducer design.

\section{DE HARVESTER DESIGN}

Fig. 3 presents the DE harvester design that follows from its mechanism of operation under the constant charge regime. Here the user's gait dictates the input mechanical pressure to the DE, including variations therein over space and time. From equation (1c), since the net energy output depends on $\lambda_{\text {init }}$ and $\lambda_{\text {final }}$, the control of the charging and discharging stages are crucial to the amount of net energy harvested, and are respectively controlled by switches 1 and 2 (Fig. 3). The mechanical and electrical behavior of the DE control the outcome of the actuation and transduction stages, and are governed by the DE's physical configuration and material properties. Electrical losses in the DE and the rectification circuitry also impact the net energy output.

Given that the net energy output of a DE harvester depends on the stretch it has incurred at the time it is charged, it follows from the spatio-temporal variance property of human gait, that an array of miniaturized DE harvesters will yield more energy than a single large DE. We propose an array of $99 \mathrm{DE}$ micro-generators spanning the shoe sole, at locations corresponding to those in foot pressure profile diagrams in Fig. 2. Energy output-maximizing control of such an array is achieved by customizing the charge timing of each DE based on the time at which maximum stretch (or maximum foot pressure) is expected at its location. In section VI-B, we will discuss a technique to achieve this in a manner that is adaptive to the interfootstep variability in a user's gait.

Individual DE harvesters should be designed to realize high transduction efficiency. Towards this end, we propose the use of the bulge configuration that will enable direct transduction and reduce mechanical losses. Fig. 4 shows a single harvester in this configuration, where DE deformation is brought about by a driver component affixed to the shoe insole. Each such harvester is comprised of a circular active area, $1 \mathrm{~cm}$ in diameter, with multiple layers of DE film that are each $5 \mathrm{~mm}$ thick and pre-stretched to $300 \%$ by $300 \%$. The harvesters that we study are made of the 3M manufactured VBH4905 acrylic DE [10].

While high transduction efficiency requires strong coupling between the force exerted by the foot and that experienced by each $\mathrm{DE}$, safe operation demands that the user's foot be isolated from the electrical activity of the DE harvesters. This is achievable with light-weight and flexible insulator materials, such as the one in [11]. The chosen transducer configuration will also impact the operational lifetime of the harvester platform. Studies of the VHB4905 DE in the proposed configuration have shown operational lifetimes of up to 22000 cycles [12], or foot steps. Further, the performance-lifetime tradeoff of DEs in an active area of research and improvements are ongoing [12] [13].

\section{DE Harvester Model}

\section{A. DE Behavior During Actuation Stage}

To analytically model DE behavior over the actuation stage, we must relate the elastic forces experienced in the DE when mechanical pressure is applied to it, or its stress, to the stretch it experiences.

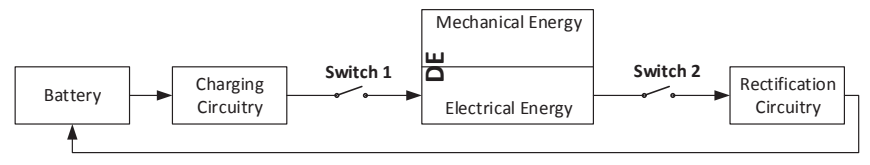

Fig. 3: Schematic of DE Energy Harvester System.

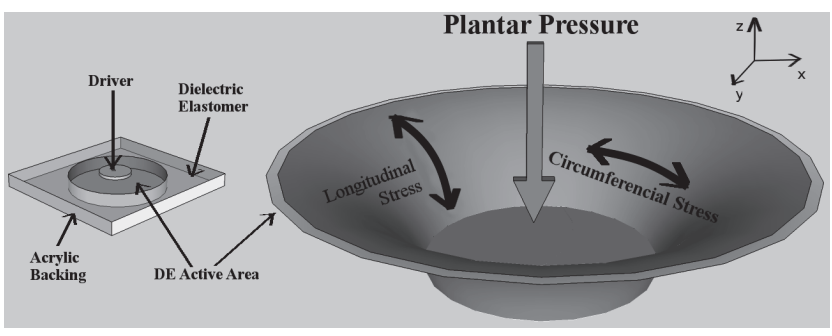

Fig. 4: DE harvester configuration.

This requires a characterization of its geometry when stretched. In contrast to the DE transduction in Fig. 1 where the DE stretch is equal and uniform along the $\mathrm{x}$ and $\mathrm{y}$ axes, the bulge configuration (Fig. 4) involves a non-uniform stretch that is also unequal in the circumferential and longitudinal directions. Although the area of the DE when stretched may be deduced via controlled measurements of its capacitance, the shape of the DE and therefore its stress may not be deduce in this manner.

We overcome this issue by numerically solving for the shape that yields minimum surface area for a given vertical displacement $h$ induced at the center of DE, by application of mechanical force via the driver. Since the net energy output depends primarily on the stretch (Equation (1d)), or change in area, a minimum area-based characterization will provide a lower bound for the net energy output. The lateral surface of the stretched DE in Fig. 4 can be deconstructed as a surface of revolution of a curve in the $\mathrm{x}-\mathrm{z}$ plane, about the $\mathrm{z}$ axis. In the x-z plane, this curve experiences boundaries at $(a, 0)$ and $(b, h)$, where $a$ and $b$ are the radii of the driver and DE active area, respectively.

Discretizing the z-axis and searching for such a curve whose shape minimizes DE surface area for a given $h$, numerically unfolds the solution. Note that this procedure benefits from significant pruning of the search space based on physical constraints on the continuity and smoothness of the curve, and the objective of minimum lateral surface area. By characterizing the geometry of a stretched DE micro-generator for a given vertical displacement $h$, we are able specify the longitudinal length $l$, the lateral surface area $A_{l a t}$, and the average cross-sectional area $A_{\text {cross }}$ of the stretched DE. Based on these values, we can calculate the average stretches $\left(\lambda_{l}, \lambda_{c}\right)$ that the DE experiences in the longitudinal and circumferential directions, respectively (Equations (2)). Further, characterizing the geometry also allows us to equate the vertical mechanical force applied to the DE by the foot, to the vertical projection of the elastic stresses induced in it, thus yielding numerical solutions to the average circumferential $\left(\sigma_{c}\right)$ and longitudinal $\left(\sigma_{l}\right)$ stresses that the DE experiences.

$$
\begin{aligned}
\lambda_{l} & =\frac{l+a}{b} \\
\lambda_{l} \lambda_{c} & =\frac{A_{l a t}+\pi a^{2}}{\pi b^{2}}
\end{aligned}
$$

As noted earlier, the behavioral model of a DE micro-generator in the actuation stage arises from a description of the relationship between the stress that the DE film experiences and the stretch that results. However, DEs are neo-hookean materials with non-linear stress-stretch relationships. As is the convention with such materials, we modeled the elastic behavior of a DE in the bulge configuration with the Ogden hyper-elastic material model [14]. To generate an accurate model, we experimentally derived the parameters of the Ogden curve for a prototype DE micro-generator. Fig. 5 shows the experimental setup for these stress-stretch measurements - A linear stage (Zaber linear stage model A-LSQ300A-E01) was used to move a driver affixed load cell (Transducer Techniques MDB Series) into 
the active area of the DE prototype that was firmly fastened in place. The displacement $h$ in the DE film was measured as the displacement in the linear stage, and the input mechanical force was measured by the load cell. A non-linear least squares fit of the measurements produced the parameters of the Ogden model specific to the proposed configuration.

For a given force applied by the foot, we can now deduce the stresses experienced by the DE. From the Ogden stress-stretch curve, we arrive at the resulting stretch and hence the vertical displacement in the DE and values of related geometrical variables.

\section{B. DE Behavior During Transduction Stage}

We outline the detailed transduction model of a DE microgenerator in the bulge configuration, which we used to compute the net energy produced. In equation (1d), whereas $\lambda_{\text {init }}$ is arrived at with the Ogden model solely from the mechanical pressure applied at the time of charging, $\lambda_{\text {final }}$ depends on the interaction between the elastic and electrostatic forces acting on the DE film throughout the transduction stage until the time at which it is discharged. To model DE behavior over the transduction stage, we modified the transduction model for uniform biaxial stretch described in [15] and addressed the geometry involved in a bulge deformation.

On charging the DE at stretch $\lambda_{\text {init }}$, the net force that leads to a further change in stretch is expressed in terms of the balance between the elastic, electrostatic, gravitational and residual mechanical pressure being applied to the DE. The equations listed below describe this "force-balance" relationship that is central to arriving at the steady-state stretch, when multiple stresses act on the DE film. It expresses the net force that leads to a change in stretch, in terms of the mass and acceleration during this change (LHS of equation below). This force is achieved as a balance between the elastic forces (first and second terms) and the sum of the electrostatic forces (third term), the gravitational forces (forth term) and additional vertical foot plantar forces (fifth term), acting on the DE. As the stretch decreases (increases), the elastic forces decrease (increase) and electrostatic forces increase (decrease), ultimately leading to a steady state.

$$
\begin{aligned}
m b \frac{\partial^{2} \lambda_{l}}{\partial t^{2}}=- & A_{\text {cross }}\left[\mu_{1}\left(\lambda_{l}^{\alpha_{1}}-\frac{1}{\left(\lambda_{l} \lambda_{c}\right)^{\alpha_{1}}}\right)\right. \\
& +\mu_{2}\left(\lambda_{l}^{\alpha_{2}}-\frac{1}{\left(\lambda_{l} \lambda_{c}\right)^{\alpha_{2}}}\right) \\
& \left.+\mu_{3}\left(\lambda_{l}^{\alpha_{3}}-\frac{1}{\left(\lambda_{l} \lambda_{c}\right)^{\alpha_{3}}}\right)\right]-2 \pi b d p^{\prime} \\
& +\frac{Q^{2}}{\pi^{2} \varepsilon_{0} \varepsilon_{r} b^{4}\left(\lambda_{l} \lambda_{c}\right)^{2}}+\frac{A_{\text {cross }} m g}{A_{\text {crossvert }}}+\frac{A_{\text {cross }} F^{\prime}}{A_{\text {crossvert }}}
\end{aligned}
$$

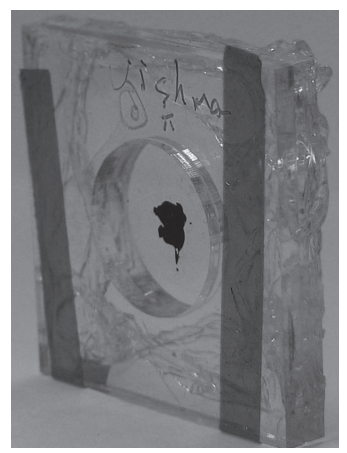

(a)

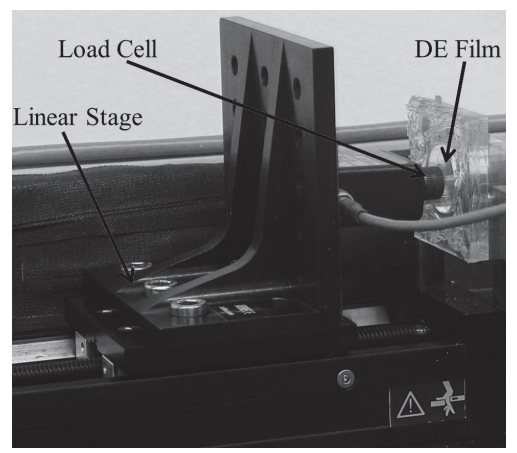

(b)
Fig. 5: (a) Prototype DE harvester, and, (b) Experimental Setup for DE prototype stress-stretch measurements.
Here, $F^{\prime}$ is the foot plantar pressure on the DE at the instant when the equation is evaluated, and $p^{\prime}$ is the residual stress in the DE due to pre-straining and is derived while fitting the Ogden model. $\mu_{p}$ and $\alpha_{p}$ are parameters of the Ogden model that capture the hyper-elastic properties of the DE. Also, $m$ is the mass of the DE, $g$ is standard gravity, $d$ is the DE thickness after it is pre-stretched but before it is operated on, $Q$ is the charge applied, and, $\frac{A_{\text {cross vert }}}{A_{\text {cross }}}$ is the fraction of the force vector that corresponds to its vertical component. This force balance equation is solved with a Runge-Kutta forth order method to produce the steady state stretch value. And $\lambda_{\text {final }}$ is the steady-state stretch value at the commencement of the discharging stage.

Given the initial stretch at the time of charging and the final stretch at the time of discharging, the net energy output $\Delta E_{D E}$ may then be derived similar to equation (1d). Whereas that equation represents equi-biaxial stretch, it can be transformed to yield the energy output for the bulge configuration by replacing $\lambda^{2}$ with $\lambda_{l} \lambda_{c}$. The net energy harvested $\left(E_{h a r v}\right)$ is proportional to the difference between the net energy output of the DE and the electrical losses incurred during charging and transduction (equation (4)). These losses $E_{\text {loss }_{\text {elec }}}$ include conduction and dielectric losses in the material, and impedancebased losses in the charging circuitry. Finally, in calculating the net energy harvested we consider the DC-DC conversion inefficiencies in the rectification circuit LossFact Lkt $_{\text {. }}$.

$$
E_{\text {harv }}=\operatorname{LossFact}_{c k t}\left(\Delta E_{D E}-E_{\text {loss }_{\text {elec }}}\right)
$$

\section{Vi. DE Harvester Array Configuration And Control OPTIMIZATION}

\section{A. Control Parameters}

Based on the DE generator operation cycle and behavior model, we identify 3 parameters that are crucial to the net energy harvested.

1) Charge Timing: As discussed in sections III-B and IV, human gait exhibits distinct spatio-temporal variance properties that we leverage via the DE 3 configuration to improve transduction efficiency. However, implicit to this argument is the fact that the DEs in the array must now be carefully and individually controlled. For example, given that the initial stretch $\lambda_{\text {init }}$ in equation (1d) is a function of the timing of the applied charge, if DEs at the toes are charged at the beginning of the stance phase when they experience no pressure from the foot, $\lambda_{\text {init }}$ will be close to 1 regardless of the foot pressure that follows at the toes during the rest of the stride. This will result in negative energy harvested due to the electrical losses. Therefore it is imperative that charge be applied as close to maximum stretch, if net energy output is to be maximized. This in-turn requires that the system accurately estimate the time at which each DE in the array will experience maximum stretch. Here, we can leverage another common property of human-gait, namely local correlation. Later in this section, we will explore an adaptive control algorithm that makes use of a few foot pressure sensor samples to enable such sn estimation.

2) Harvester Thickness: A thicker DE film can withstand much more pressure without rupture, while a thinner one will stretch more at comparable applied pressures. In other words, thinner DE films can harvest more energy at a given pressure that thicker films, as long as the pressure is acceptable. Fig. 6 plots the stress-stretch data as well as the best fit curves for our experimental measurements for 3 variations of the bulge configurations, one with 3 DE layers, a second with 6 layers and a third with 9 layers. It is apparent that a configuration with more layers must experience more stress to achieve similar levels of stretch. Given this observation, the spatial variance property of human gait can enhance net energy yield. To increase the energy output at low input pressure regions of the foot, such as the arches, we propose an adaptive harvester placement strategy. Here, 
the thickness of a harvester in the array will depend on the amount of pressure observed at its location. Based on the maximum pressure observed over a training dataset for each DE location, decisions will be made to fit high pressure locations with thicker harvesters and low pressure locations with thinner ones. Specifically, to address harvester lifetime and user-comfort issues, a location is occupied by the thinnest harvester for which the maximum observed pressure over the training dataset produces no more than a vertical displacement of $9 \mathrm{~mm}$, an acceptable level of compression for soft cushioned shoes [16].

3) Applied Voltage: Equation (1d) suggests that higher applied voltages should yield higher net energy outputs, ad infinitum. However, since the applied voltage is related to the applied charge, adding too much charge to the elastomer film will create large electrostatic forces on it, which will prevent full relaxation of the film at the end of the stance phase. As $\lambda_{\text {final }}$ is driven away from 1 at higher applied voltages, extremely high voltages are undesirable. Fig. 7 shows the net energy output predicted by the DE behavior model when a harvester in the proposed configuration is exposed to different input pressures and charged over a range of voltages. We observe that the output energy is not a monotonically increasing function of the applied voltage. As a result, we restrict system operation to $8000 \mathrm{~V}$, the voltage at which the net energy output, averaged across input pressures studied, is the maximum. Note that the plot also factors in the maximum pressure-specific DE thickness configuration described above. For this reason, the output energy does not monotonically increase with input pressure. The energy output can be separated into 3 bands of input pressure, such that the net energy output increases with the applied pressure within each band. Here, the lower pressure bands are governed by thinner harvesters, and sharp transitions in the net energy output occur as the input pressure surpasses the usercomfort / harvester lifetime thresholds for the thinner harvester. This necessitates switching to a thicker harvester leading to a sharp decline in energy output across adjacent bands.

\section{B. Control of Harvester Array in the Charging Stage}

As discussed earlier, the transduction efficiency and net energy output is significantly enhanced by individually controlling the charge times of DEs in the array. In order to accurately predict the optimal charge timing of each harvester, we leverage the local correlation property of the foot pressure profiles. This property enables nearoptimal estimation at most of the 99 DE harvesters based on just a few pressure sensor samples, thereby yielding near-optimal aggregate net energy output at a small additional energy cost (for the pressure samples).

Multiple approaches may be conceptualized for the inclusion of foot pressure sensors into the system. The DEs may be juxtaposed

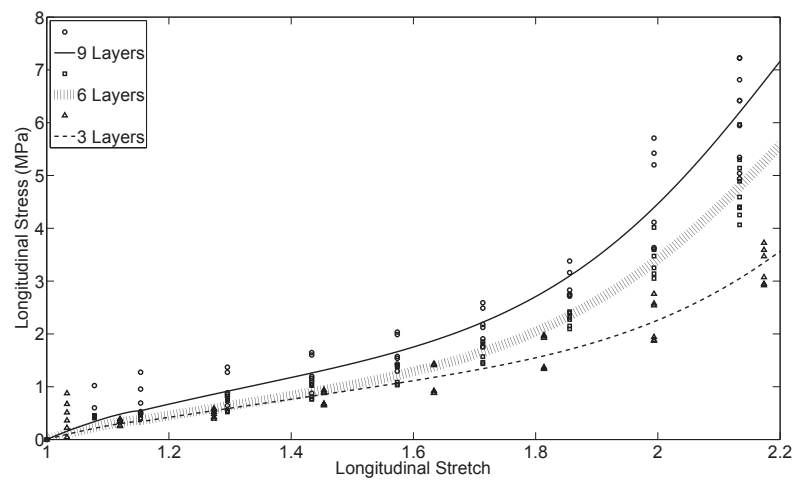

Fig. 6: Experimentally collected measurements for the stress-stretch relationships of DE harvesters prototypes at different number of layers, and the corresponding best fit curves. with passive-resistive sensors, or, the DEs may support integrated or dual-mode operation as sensors or generators. Sensor mode operation of DEs may consume up to a few $\mathrm{nJ}$ more than passive-resistive sensors, but not more; Since our goal is not to maximize energy output when they are operated as sensors, DEs may be charged at a low voltage to measure a change in capacitance and calculate the applied pressure therefrom. If the DE is already charged for harvesting, the changes in its capacitance can still be measured in a similar manner.

We formally define the charge timing prediction problem as follows. We assume a maximum of $T$ epochs between the start and end of the stance phase, over which we are given the foot pressure readings at each harvester location. We would like to predict the time at which maximum pressure is observed at each location in a manner that maximizes the net system energy output. Here the net system energy output is computed as the net harvester output (equation (4)) summed over all harvester, minus the energy cost of each pressure sample used in the charge timing prediction. Further, the following constraints apply:

- If the optimal charge timing for a DE is predicted as epoch $k$, the prediction must be made between epochs 1 and $k-1$.

- If the optimal charge timing for a DE is predicted as epoch $k$, the prediction must be based on samples taken solely between 1 and $k-1$.

- There is a maximum number of samples that may be used at each epoch, which may be different for different epochs. This constraint is governed by the battery power rating and the power draw of the target application that we are trying to power. However, samples taken for the purposes of the target application may also be applied to charge timing prediction.

1) Charge Timing Prediction: We take a statistical approach to solving the charge timing prediction problem - Given a training dataset, we apply a semi-parametric survival analysis based technique to predict optimal charge timing. Originally developed to predict machine failure times and the death of biological organisms, their use has expanded to several applications requiring time-to-event prediction. The survival rate $S(t)$ of a harvester is the probability that its optimal charge timing hasn't yet occurred at epoch $t$. The hazard rate $f(t)$ is the conditional probability that the optimal charge timing will occur at epoch $t$, under the condition that it hasn't occurred yet. The cumulative hazard rate $F(t)$ is the sum of the hazard rates less than or equal to $t$. And from equation (5a), where $\Delta t$ is the duration of an epoch, it follows that $F(t)$ approaches the negative log of the

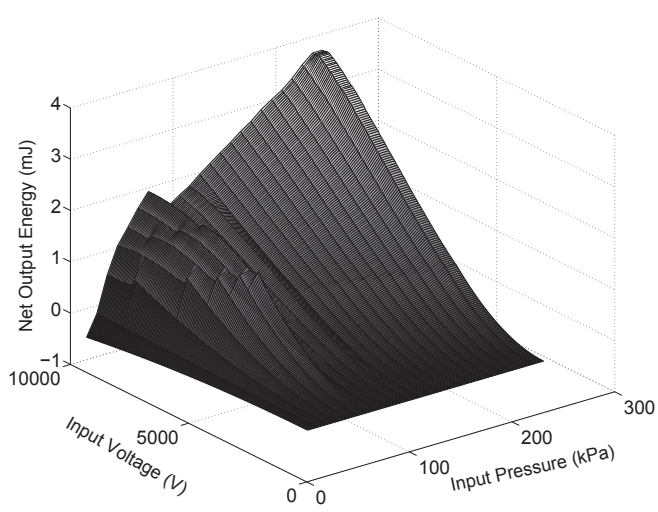

Fig. 7: Net Energy Output $\left(\Delta E_{D E}\right)$ of harvester model for different input pressures and applied voltages. 
survival rate as $\Delta t$ approaches 0 .

$$
\begin{aligned}
f(t) & =\frac{S(t)-S(t+\Delta t)}{\Delta t S(t)} \\
F(t) & =\sum_{t^{\prime}=0}^{t} f(t) \\
& =-\log (S(t))
\end{aligned}
$$

Cox regression, $\operatorname{cox} \operatorname{Reg}()$ is a semi-parametric survival regression technique. Given a dataset of optimal charge timing for a DE over several steps, and a set of covariates $x_{1}$ thru $x_{n}$, that signify a subset of samples, the algorithm constructs a cumulative hazard rate function based on a non-parametric baseline estimate of the function $\left(F_{0}\right)$, and multiplicative effects parameter composed of a linear combination of the samples $x_{i}$. The coefficients $\beta_{i}$ are derived as maximumlikelihood estimates for on the training set.

$$
F(t)=F_{0}(t) e^{\beta_{1} x_{1}+\beta_{2} x_{2}+\ldots+\beta_{n} x_{n}}
$$

Algorithm 1 outlines the procedure used to decide whether a DE should be charged at the following epoch based on the samples available so far. The algorithm is called for each uncharged harvester and at each epoch, and is provided the samples available thus far, along with the corresponding cox regression parameters. With these inputs, the algorithm computes the cumulative hazard function for the following epoch $k$ and converts the function to the corresponding survival rate function $\mathrm{Sr}$. While the survival rate function tells us the probability that the optimal charge timing won't have occurred at each future epoch, lines 3 thru 5 of the algorithm convert this to the probability that optimal charge timing will occur at each future epoch. If the highest probability co-incides with the following epoch, a decision is taken to charge the DE. The algorithm waits until just before the optimal charge timing is expected for two reasons. First, this provides additional information that is likely to lead to a better decision. Second, we observed that with fewer epochs remaining until the end of the stance phase, it increases the kurtosis of the optimal charge timing probability density function thereby improving our expectation.

2) Offline Optimization: Given our use of the survival analysis technique, the charge timing prediction problem stated in section VI-B turns into a sample selection problem that offers the best predictions while abiding by the constraints therein. We solve the problem with the stepwise-regression based optimization in algorithm 2. In the absence of samples, each harvester's timing is predicted from its baseline hazard functions. Else, each sample, $s_{j}$, is evaluated via $\operatorname{avg} \operatorname{Imp}()$ that measures the improvement $s_{j}$ affords to the prediction of harvester $h_{i}$ (line 9), averaged over training steps. This improvement contributes to $s_{j}$ 's profit $s p_{j}$ (lines 10 thru 12).

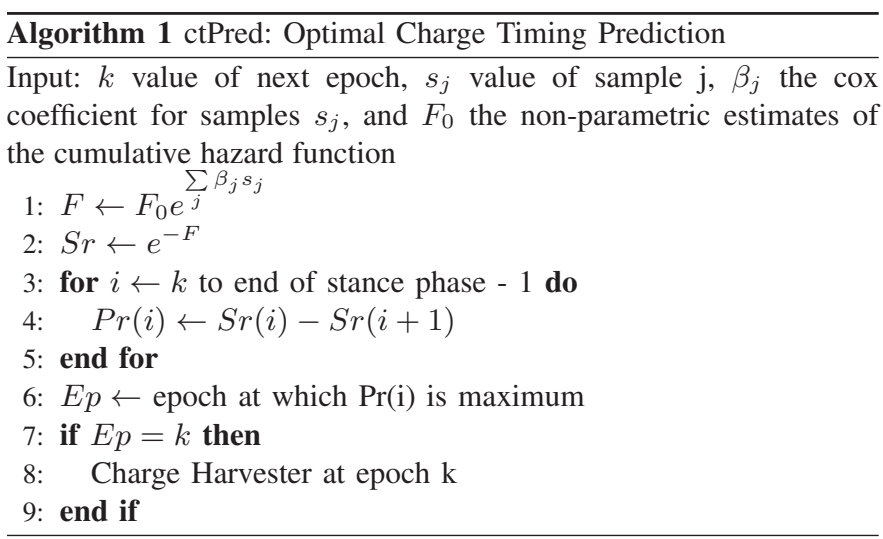

The prediction's improvement is measured in terms of the increase in energy output of $h_{i}$ due to the addition of $s_{j}$ into its predictive sample subset for epoch $k$. At each iteration, the most profitable sample is selected into the predictive subset (lines 14 thru 17). Note, that if a sample is available freely, due to its necessity in the target application, its cost $C_{j}$ is 0 . Otherwise, its cost corresponds to the energy expended in acquiring the sample. Power constraints are observed by line 19, and overfitting is prevented by applying the Akaike Information Criterion (AIC) to evaluate goodness of fit before accepting a sample into a harvester's predictive subset for an epoch (lines 6 thru 8). For the sake of brevity, we have implicitly assumed that lines 11 and 18 include mechanisms to denote which harvester and epoch each included sample will be used to predict.

\section{Control of Harvester Array in the Discharging Stage}

Aggregate net energy output depends on the amount of stretch experienced by the DE harvesters. However, all DEs in the array experience stretch solely during the stance phase, when the foot is in contact with the ground. Hence, the $T$ epochs over which DE charging control is actualized begin at the start of the stance phase and end when the stance phase ends. Towards this end, we assume the application of one of the techniques discussed in [17] to identify the transition between the stance and swing phases with a few sensors. By detecting the transition between phases, we are also able to achieve discharging stage control. In other words, all DEs may be simultaneously discharged at the end of the stance phase. Drawing from the design in [18], we have also designed the rectification circuitry for the system that will combine this simultaneously discharged energy output before sending it towards the energy store or battery. Although we exclude its description for brevity, we highlight salient facts that help quantify the rectification

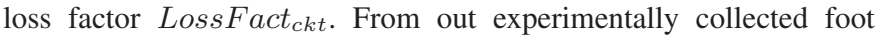
pressure datasets, we expect the total mechanical energy expended by foot-strikes to exhibit low variance. Therefore, the DC-DC converter

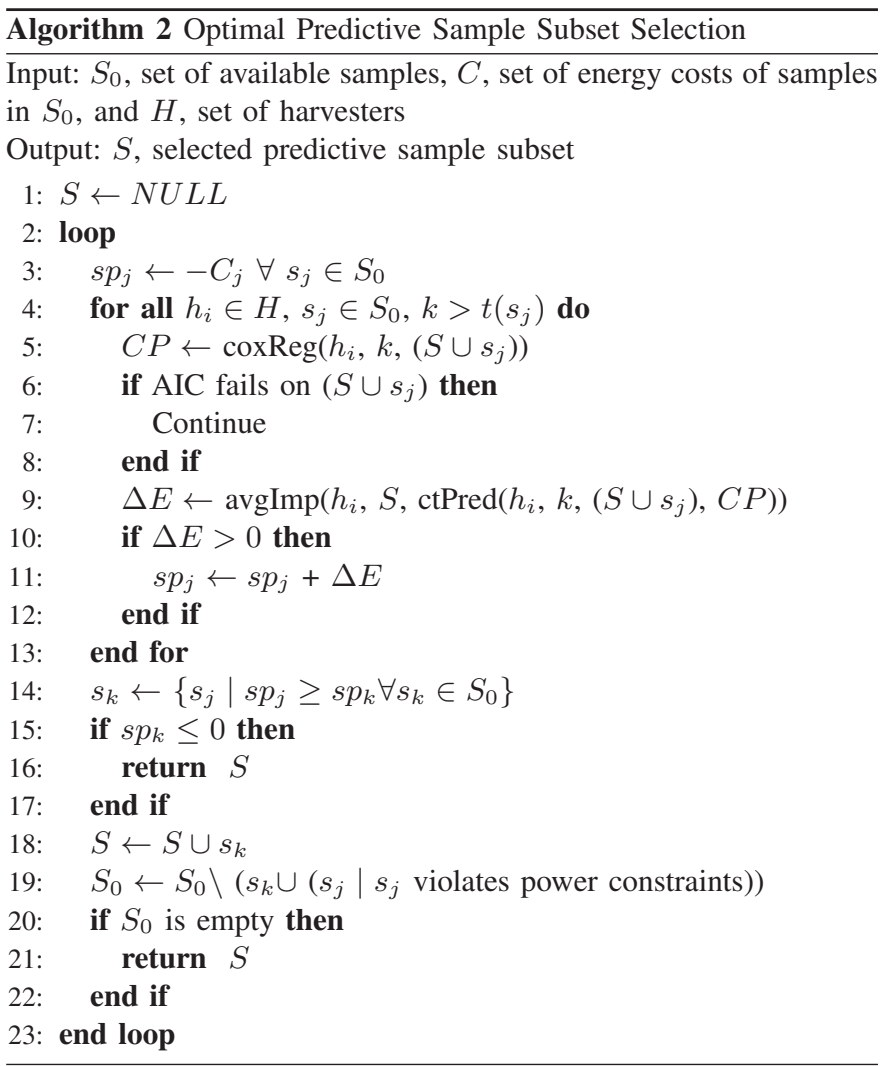


used for rectification can be designed to achieve high efficiency over the narrow expected output current range for a user. It has been shown that under low input current variance, DC-DC converters can be designed to yield efficiencies upwards of $80 \%$ [19]. Therefore, we set the rectification loss factor to 0.8 .

\section{Electronic Control}

The harvester platform described thus far requires electonic control during the charging and discharging stages of DE operation. Towards this end we leverage the design of Hermes [6], a human balance monitoring system that measures plantar pressure. Hermes employs the low-power MicroLEAP platform [20], which provides a multiplexed 8-channel 16-bit ADC, 8Mbits of flash memory, and an MSP430 micro-controller. This low-power electronic control platform will be used to control switches 1 and 2 in Fig. 3 based on the control algorithms described above.

\section{Performance Evaluation}

We evaluate the system performance with experimentally collected foot plantar pressure datasets by Hermes from 3 subjects. Each subject is represented by 2 datasets, one for each foot. Two of the three subjects are lighter individuals (one male and one female) who expend less mechanical force with their foot-strikes. The third subject is a heavier male individual. Each of the datasets offer several steps worth of data at each of the ninety-nine harvester locations. This allows us to derive performance at all harvesters and apply the predictive sample subset selection and control algorithms, thereby evaluating the net harvested energy. We divide each dataset into a training subset comprised of $80 \%$ of the data and a testing subset comprised of the rest.

Fig. 8 presents the maximum energy scavengable (with perfect charge timing prediction), averaged over the strides in the datasets, for both feet of a single subject. Here, the maximum achievable output with and without the location-specific thickness configuration are juxtaposed. While the areas that normally experience large amounts of plantar pressure produce exactly the same amounts of energy, surrounding areas, that were previously anemic in their output begin to look more promising.

To test the predictive sample subset selection algorithm, we leveraged the research in [17] towards power-constrained sub-sampling with Hermes. The authors therein, proposed a semantic accuracy preserving sampling strategy that reduced the energy consumption of Hermes from $72 \mathrm{~mJ}$ to $43 \mathrm{~mJ}$ per stride, under power constraints of at most 5 sensors sampled at a time. We leverage the output of the algorithm proposed in that work and constrained our algorithm by those samples. Additional samples were allowed to be chosen,

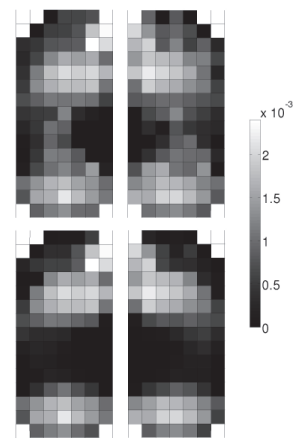

Fig. 8: Maximum energy harvestable ( $\mathrm{J}$ ) from the left and right feet of a subject in the plantar pressure dataset. Array with uniformly thick DEs (bottom), and with location sensitive DE thickness (top). if a power constraints of 5 samples per epoch permitted this. The following analysis was conducted under these constraints.

Fig. 9 shows the energy scavenged based on our proposed sample subset selection and adaptive DE charge timing control algorithms for the dataset corresponding to the left foot in Fig. 8, and compares it to the maximum energy scavengable. On average, our proposed system can produce over $80 \mathrm{~mJ}$ per foot strike. The location-sensitive DE thickness configuration boosts this by another 20-26\%. However, some of the steps in both the training and testing datasets are a result of the subject turning around rather that walking. Such steps produce less foot plantar force, and are infrequent thereby yielding insufficient data for the training algorithms. Owing to lower amounts of transducable energy and poorer prediction performance over such steps, the net energy harvested drops. It is noteworthy that due to our algorithms inbuilt penalties for overfitting, the loss of energy due to imperfect charge timing does not vary disceribly from the training subset (first $80 \%$ of steps) to the testing subsets.

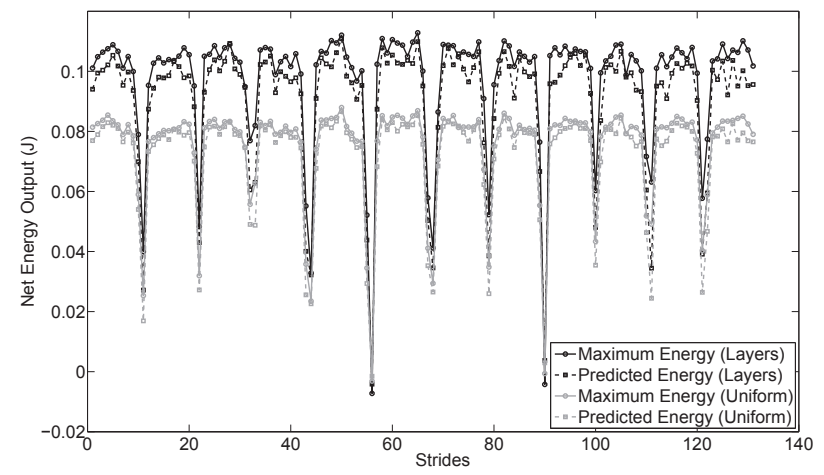

Fig. 9: Maximum possible vs. achieved aggregate net energy output, averaged over the testing subsets of a dataset. Plots compare output for uniform DE thickness to location sensitive DE thickness.

To better quantify the degree to which charge timing is imperfect we analyzed the impact of our sampling subset selection algorithm. In Fig. 10, we compare the performance of 4 sampling subset selection techniques over the testing subset: (i) Our proposed technique, (ii) an adaptive system where all available samples are provided to the coxregression algorithm, (iii) an algorithm that estimates charge timing of each harvester as the median epoch, over the training dataset, at which its net energy output was maximum, and, (iv) an algorithm that charges all DEs in the array at the same epoch, which is estimated as the median epoch, over the training dataset, at which the net energy output aggregated over all DEs was maximum. The comparison is shown for all 6 datasets. It is clear that an adaptive system can

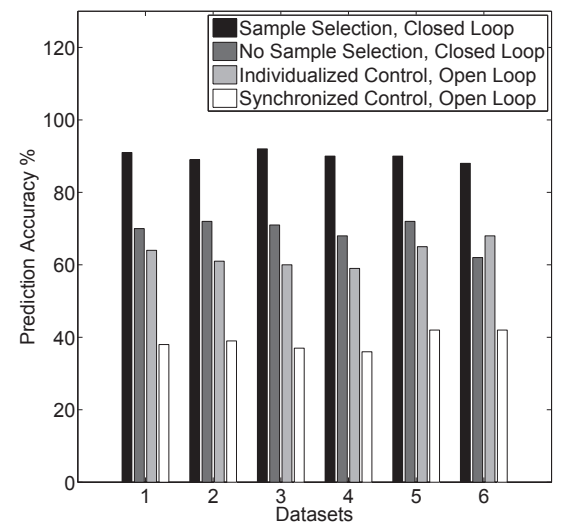

Fig. 10: Bar Plots for the prediction accuracy of different sample selection and optimal charge timing prediction techniques over the testing subsets of each of our datasets. 
yield more energy. Furthermore, the prediction accuracy of the second technique over the training subset was more than $98 \%$ for each of the datasets. However, the accuracy over testing does not rise beyond $75 \%$, clearly a case of overfitting. A comparison of the third to the forth technique highlights the merits of individualized control over the array. Leveraging the spatio-temporal variance property of the plantar pressure profile significantly enhances net energy output.

Table I compares the average aggregate energy output of our proposed system, under both DE configurations (with and without location-specific thickness), for each of the datasets. Owing to the superior material properties of DEs and the accuracy of our algorithm, our system outperforms the piezoelectric-driven system in [3] that produced $20 \mathrm{~mJ}$ per stride. Also, across our datasets and with both configurations, we are able to satisfy the energy demands of our target applications $(43 \mathrm{~mJ})$. Further, owing to our algorithm's penalties for overfitting, we observed minimal difference between performance over the training and testing subsets for all datasets $(<1 \%)$.

TABLE I: Comparison of Foot Strike Energy Harvesting Output.

\begin{tabular}{|c|c|c|}
\hline $\begin{array}{c}\text { Scavenger } \\
\text { Mechanism }\end{array}$ & $\begin{array}{c}\text { Net Energy } \\
\text { Output (mJ) } \\
\text { - Layers }\end{array}$ & $\begin{array}{c}\text { Net Energy } \\
\text { Output (mJ) } \\
\text { - Uniform }\end{array}$ \\
\hline Subject 1, Left & 107.1 & 91.5 \\
Subject 1, Right & 120.5 & 98.3 \\
Subject 2, Left & 86.9 & 68.0 \\
Subject 2, Right & 81.9 & 69.5 \\
Subject 3, Left & 90.0 & 71.8 \\
Subject 3, Right & 80.8 & 71.2 \\
\hline
\end{tabular}

We also sought to answer whether there was something inherent in the samples afforded by the algorithm in [17] that yielded such high outputs. The answer is yes. Fig. 11 shows the prediction accuracy from using between 1 and 5 samples per epoch restricted to the samples produced by [17]. It also shows the prediction accuracy when the samples are randomly chosen at each epoch. Note that the sample subset selection algorithm was run on the chosen samples. Both curves correspond to averages across 3 datasets. The dashed curve in Fig. 11 shows that a few random samples per epoch are insufficient to produce accurate predictions. Here, the prediction accuracy depends on the baseline hazard function as no samples are chosen into the predictive subset. As the number of samples per epoch increases beyond 12 , the results improve to recognizable levels!

\section{CONCLUSION}

We have proposed the use of a novel transducer technology to scavenge energy from human foot-strikes and power wearable systems, in an effort to resolve lifetime and maintenance issues. We study

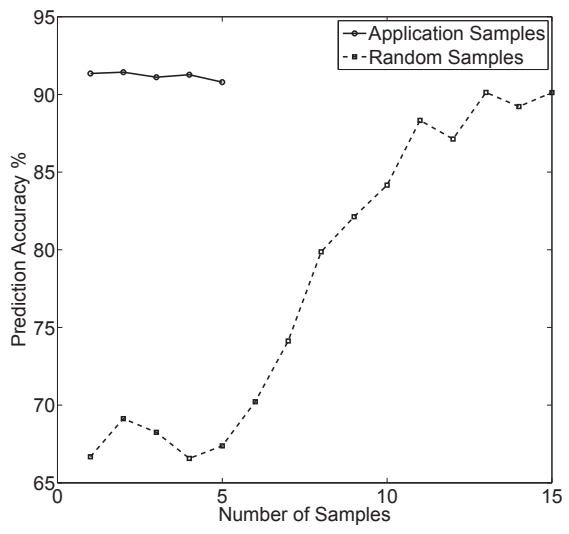

Fig. 11: Performance of our propose predictive sample subset selection algorithm under different power constraints. adaptive configuration and dynamic system control techniques, based on user gait characteristics, to maximize the platform's net energy output while addressing user comfort issues. We have characterized and modeled the transduction behavior of the technology, and applied this model to experimental plantar pressure datasets to show that the design can yield up to $120 \mathrm{~mW}$ from walking subjects.

\section{REFERENCES}

[1] I. Beretta, F. Rincon, N. Khaled, P. Grassi, V. Rana, D. Atienza, and D. Sciuto, "Model-based design for wireless body sensor network nodes," in 13th Latin American Test Workshop (LATW), 2012, pp. 1-6.

[2] M. Vallejo, J. Recas, and J. L. Ayala, "Channel analysis and dynamic adaptation for energy-efficient WBSNs," in Proceedings of the 6th International Conference on Ubiquitous Computing and Ambient Intelligence. Berlin, Heidelberg: Springer-Verlag, 2012, pp. 42-49.

[3] J. A. Paradiso and T. Starner, "Energy scavenging for mobile and wireless electronics," IEEE Pervasive Computing, vol. 4, no. 1, pp. 1827, Jan. 2005.

[4] P. Mitcheson, E. Yeatman, G. Rao, A. Holmes, and T. Green, "Energy harvesting from human and machine motion for wireless electronic devices," Proceedings of the IEEE, vol. 96, no. 9, pp. 1457 -1486, sept. 2008.

[5] R. D. Kornbluh, R. Pelrine, H. Prahlad, A. Wong-Foy, B. McCoy, S. Kim, J. Eckerle, and T. Low, "From boots to buoys: promises and challenges of dielectric elastomer energy harvesting," Polymer, vol. 7976, no. 1, 2011.

[6] H. Noshadi, S. Ahmadian, H. Hagopian, J. Woodbridge, F. Dabiri, N. Amini, M. Sarrafzadeh, and N. Terrafranca, "HERMES - mobile balance and instability assessment system," in Proceedings of BIOSIGNALS, 2010, pp. 264-270.

[7] E. Costanza, S. A. Inverso, E. Pavlov, R. Allen, and P. Maes, "eyeq: Eyeglass peripheral display for subtle intimate notifications," in Proceedings of Mobile HCI, 2006.

[8] T. McKay, B. OBrien, E. Calius, and I. Anderson, "Self-priming dielectric elastomer generators," Smart Materials and Structures, vol. 19, no. 5,2010

[9] T. A. Gisby, E. P. Calius, S. Xie, and I. A. Anderson, "An adaptive control method for dielectric elastomer devices," Proceedings of SPIE Electroactive Polymer Actuators and Devices, vol. 6927, pp. 69271C$69271 \mathrm{C}-8,2008$.

[10] 3M, "3M VHB TM tapes," Technical Data Sheet, June 2011.

[11] U. Liepold, "Isolation material for electrical components is a foam ceramic produced as a formed housing," German Patent Application DE10 147980 A1, April 30, 2003.

[12] J. Lucking Bigu, P. Chouinard, S. Proulx, G. Miron, and J. Plante, "Preliminary assessment of manufacturing impacts on dielectric elastomer actuators reliablity," in Proceedings of Smart Materials and Structures, 2009, pp. 303-314.

[13] W. Yuan, P. Brochu, H. Zhang, A. Jan, and Q. Pei, "Long lifetime dielectric elastomer actuators under continuous high strain actuation," Electroactive Polymer Actuators and Devices (EAPAD), vol. 7287, pp. 72 8700-72 8700-8, 2009.

[14] R. W. Ogden, "Large deformation isotropic elasticity - on the correlation of theory and experiment for incompressible rubberlike solids," Proceedings of the Royal Society of London. Series A, Mathematical and Physical Sciences, vol. 326, no. 1567, pp. 565-584, 1972.

[15] C. Jean-Mistral, S. Basrour, and J.-J. Chaillout, "Modelling of dielectric polymers for energy scavenging applications," Smart Materials and Structures, vol. 19, no. 10, p. 105006, 2010.

[16] Optimal performance index system. [Online]. Available: http://www. opistest.com/

[17] V. Goudar and M. Potkonjak, "Power constrained sensor sample selection for improved form factor and lifetime in localized bans," in Proceedings of the 3rd Conference on Wireless Health, 2012.

[18] G. Ottman, H. Hofmann, A. Bhatt, and G. Lesieutre, "Adaptive piezoelectric energy harvesting circuit for wireless remote power supply," IEEE Transactions on Power Electronics, vol. 17, no. 5, pp. 669-676, 2002.

[19] T. Simunic, L. Benini, and G. De Micheli, "Energy-efficient design of battery-powered embedded systems," IEEE Transactions on Very Large Scale Integration (VLSI) Systems, vol. 9, no. 1, pp. 15-28, 2001.

[20] L. Au, W. Wu, M. Batalin, D. Mclntire, and W. Kaiser, "Microleap: Energy-aware wireless sensor platform for biomedical sensing applications," in Proceedings of Biomedical Circuits and Systems Conference (BIOCAS), nov. 2007, pp. $158-162$. 